\title{
Win at Any Cost: Examining Personality Correlates of Achievement in Adolescent Athletes
}

\author{
Winston Park ${ }^{1}$ and Matthew Herman" \\ ${ }^{1}$ Gillman School, Baltimore, MD, USA \\ "Advisor
}

$\underline{\text { ABSTRACT }}$

Achievement in sports is often attributed to being highly competitive or doing "whatever it takes" to win as opposed to being agreeable. The current consensus is that these traits, manifested as hypercompetitiveness, Machiavellianism, and agreeableness, tend to be highly related within adult populations and athletes. Machiavellianism, the "whatever it takes' ' attribute and hypercompetitiveness have been found to be positively correlated with one another while both are negatively correlated with agreeableness. Previous studies have failed to examine the same phenomenon in individual-sport adolescent athlete populations, in addition to how these trends may influence athletic achievement. In this study, a random sampling of an adolescent athlete population was given a battery of personality scales: The Hypercompetitive Attitude Scale, the MACH-IV, and an IPIP Agreeableness Measure. The participants' highest national rankings in their sport were also examined. Previous findings in adult populations were replicated in this study, as Machiavellianism and hypercompetitiveness were found to have a strong negative correlation with agreeableness $(\mathrm{r}=$ $-0.678, \mathrm{p}=0.000039$, and $\mathrm{r}=-0.690, \mathrm{p}=0.000025$, respectively). Machiavellianism displayed a moderate, positive correlation with hypercompetitiveness $(r=0.496, \mathrm{p}=0.005312)$. However, the differences in Machiavellianism and hypercompetitiveness between those who had achieved a rank of at least top 15 in the country and other participants were not found to be statistically significant. These results show that athletic achievement in adolescents was not significantly affected by the personality traits of Machiavellianism and hypercompetitiveness. Additional research is needed to discover what personality traits constitute a champion.

\section{Introduction}

Many have claimed that there is a "winning personality" associated with creating champions at a young age. Adolescence especially is a time where competitive activities such as sports are selected to be pursued to a higher level. People in this demographic are in the process of discovering and honing their talents as their brains prune unnecessary neurons to prioritize the skills they focus on(Siegel, 2015). Those who are able to develop this affinity often compete against their peers with the hopes of becoming a professional or getting recruited by a top institution. Small individual sports are especially competitive, as there are few recruiting spots and team camaraderie while players form rivalries to climb the national ranks. The epitome of such a hypercompetitive environment is the sport of squash. According to the US Squash organization, squash boasts around 1.2 million players in the US. Yet, only those who rank around the top 100 spots in the age division have any hope of being noticed by university teams, which are offering fewer and fewer spots each year(Barrett, 2020). Because of this, adolescent squash athletes are the perfect group for the examination of what exactly constitutes a winner. In an unforgiving ranking system and a small community where tensions are high, what is the difference between those who rise to the top versus those who do not? 


\section{Hypercompetitiveness}

The trait of hypercompetitiveness certainly seems like a good place to start. Hypercompetitiveness was conceptualized from psychoanalyst Karen Horney's neurotic profile of moving against others(Horney, 1937). Horney's theory of neurosis details the hypercompetitive neurotic as someone who constantly seeks to be seen controlling and attacking those around them. When they fail to exhibit superiority, intense shame and embarrassment ensues. Thus, the trait consists of constantly looking to dominate others even in noncompetitive activities.

In order to measure hypercompetitiveness, researchers have developed a Hypercompetitive Attitude Scale that has since been empirically validated in adult and adolescent populations(Ryckman 2011 \& Menesini 2018). The Hypercompetitive Attitude Scale consists of 26 questions on a 5-point Likert scale that require the subject to respond to prompts such as "Winning in competition makes me feel more powerful as a person". A selected response of Strongly Disagree would score low in Hypercompetitiveness(one point) and an answer of Strongly Agree would score the highest(five points), The scale additionally features reverse-scored questions that serve as a mechanism against "always agree" in which the subject circles the same option(typically agree) for all questions without taking the time to answer honestly. An example of a reverse-scored question on the Hypercompetitive Attitude Scale is "I do not see my opponents in competition as enemies". An answer of Strongly Agree in this case would yield the lowest score of one point, while answering Strongly Disagree is worth five. Reverse-scored questions therefore maintain the integrity of the scale.

While research has tackled the question of how hypercompetitiveness influences work performance among adults, few have examined the relationship between hypercompetitiveness and athletic performance in youth(Yearick, 2018). When hypercompetitiveness has been investigated in athletes, it is often paired with the trait of Machiavellianism.

\section{Machiavellianism}

The personality trait of Machiavellianism has commonly been associated with the traits of narcissism and psychopathy in the infamous Dark Triad(Paulhus and Williams 2002). These traits were linked by a pattern of antisocial and selfish behavior. However, Machiavellianism is a multidimensional trait that is capable of being assessed independently of the other Dark Triad traits(Rogoza and Cieciuch, 2020). The trait of Machiavellianism was first identified by researchers Christie and Geis, who derived it from core themes from the writings of the Renaissance philosopher Niccoló Machiavelli. The three main themes were then implemented as the dimensions of Machiavellianism: lack of morality, cynicism, and utilitarianism. These dimensions are present in the MACH-IV scale, a self-report measure which was designed to properly assess all facets of Machiavellianism(Christie and Geis, 1970). The scale presents 20 items on a five-point Likert scale. Questions reflect the dimensions of Machiavellianism through regular and reverse-scored items such as "People suffering from incurable diseases should have the choice of being painlessly put to death."(utilitarianism), "Most people are brave"(reverse-scored, cynicism), and "It is wise to flatter important people"(lack of morality). While the MACH-IV was originally meant for use in adult samples, it has been used reliably in empirical research centered around adolescent sample populations(Láng and Birkás, 2015).

Machiavellianism has been a subject of increasing interest within the context of competition. Various researchers have attempted to examine the "competitive personality" using the Dark Triad. One study on adult Spanish athletes conducted by González-Hernández, Cuevos-Campos, and their team revealed a positive correlation in competitive attitude and the Dark Triad traits. Specifically, Machiavellianism was shown to have a positive relationship to how one perceives their competitive environment and their own sense of failure(González-Hernández and CuevosCampos, 2020). A heightened feeling of failure and an environment characterized by unceasing hostility are characteristic of hypercompetitiveness. Studies seeking to establish a link between Machiavellianism and hypercompetitiveness, while few in number, have generally uncovered a positive relationship in adult populations(Houston and Queen, 
2015; Ryckman and Thornton, 1994). Individuals who score high in Machiavellianism, or "high Machs", have been demonstrated to have higher rates of cheating in competitive environments(Cooper and Peterson, 1980). It appears that not only do Machiavellianism and hypercompetitiveness tend to occur together, but that they can be influenced by a highly competitive environment as well.

\section{Agreeableness}

Agreeableness is one of the Big Five personality traits introduced by McCrae and Costa, who derived the Five Factor Model of personality from Allport's lexical analysis of 4,500 words for personality(Vinney, 2018; Cherry, 2019). The Big Five personality traits have been shown to influence various factors across a person's life, including physical and mental health, education, political orientation, etc.(Soldz, 2002). Besides agreeableness, the other Big Five personality traits are Conscientiousness, Extraversion, Neuroticism, and Openness to Experience.

The trait of agreeableness itself consists of six dimensions: trust, morality, altruism, cooperation, modesty, and sympathy. Therefore, people who score high in agreeableness are generally kind and helpful. agreeableness is typically measured along with the other Big Five traits in the NEO Personality Inventory-Revised, a self-report scale consisting of items that measure each Big Five trait with items that assess all of their dimensions(McCrae and Costa, 2005). Items that reliability measure agreeableness are also found in the International Personality Item Pool based on Johnson's 120-item NEO personality inventory within the public domain(Johnson, 2014). These items, similar to the NEO PI-R, assess each of the six dimensions of agreeableness. For example, an item within the dimension of Trust is "I believe others have good intentions". Each item is graded on a 5-point Likert scale, and there are reverse-scored items present, in which a response of "Strongly Agree" would result in one point instead of five, and vice versa.

Given that agreeableness is characterized by trust, cooperation, and morality while Machiavellianism features lack of morality and cynicism, it is no surprise that numerous literature indicates a negative relationship between the two. One study investigating Machiavellianism through the lens of the Five Factor Model discovered that all Dark Triad traits displayed a negative correlation with all facets of agreeableness(DeShong and Helle, 2017). Agreeableness was additionally found to be negatively correlated with hypercompetitiveness in another study examining a sample of young adults(Ross and Rausch, 2003). However, no studies have evaluated the strength of the relationships between these traits in a sample of individual-sport athletes.

The first objective of this study aims to assess the correlation between the personality traits of Machiavellianism, agreeableness, and hypercompetitiveness in adolescent individual-sport athletes. This study predicts that hypercompetitiveness will have a strong positive relationship with Machiavellianism and a negative relationship with agreeableness, while Machiavellianism and agreeableness will share a strong negative correlation. The high-strung, competitive environment that comes with an individual sport such as squash is predicted to exaggerate the correlations between the traits discovered in previous literature. In addition, the adolescent population may be more competitive from the added pressures of college and the US Squash ranking system. In a situation with higher stakes, the drive to "do anything to win" may be significantly increased. The second objective of this study is to determine how the presence of hypercompetitiveness, agreeableness, and Machiavellianism influences achievement in competition. Thus, the second hypothesis of this study predicts that individuals who have reached higher all-time US squash national rankings will have significantly higher hypercompetitiveness and Machiavellianism scores than those who have not. 


\section{Methods}

\section{Participants}

Participants were randomly sampled from college squash camps run by Collegesquashcamps.com across a period of six weeks- each camp lasted one week and had different participants. Five participants were selected from each camp, resulting in thirty total participants $(\mathrm{N}=30) .22$ males and eight females participated in this study. 11 of the participants identified as East Asian American, two participants were East Asian but had nationalities outside of the US(Hong Kong and Malaysia), eight Caucasian Americans and two participants who identified as both East Asian American and Caucasian American. Three self-identified as Indian-American, one was Indian of a different nationality(Singapore), one was African American, and two identified as Palestinian/Jordinian. The camps were selected as a site for recruiting participants because each camp had a diverse representation of squash players from across the United States. If any player who had already participated in the study was randomly selected again(only possible if they were participating in multiple camps), they would be removed from the pool and another participant would be randomly selected. In order to be put into the random sampling pool, participants must have been a squash player, had a public US Squash profile, and been between the ages of 10-25 years of age(the range of the adolescence period). All participants were fully informed of the procedure and aims of the study. They were informed of the risks and ethical standards that the study was required to uphold, and were encouraged to contact the Ethics Office of the American Psychological Association should they have any complaints or further inquiries. Participants were informed of their right to refuse or renege on the study at any time, and were provided an incentive of a five dollar donation to a charity of their choice. Participants were all asked to sign an informed consent form. For participants under 18 years old, their parent or guardian signature was also procured.

\section{Constructing an Agreeableness Scale}

In order to create a unique scale that solely measured agreeableness, items were taken from Johnson's IPIP-NEO-120. The scale featured thirty empirically validated items from the IPIP-NEO-120, with five items on a five-point Likert scale from each dimension of agreeableness. Both regular and reverse-scored items were implemented within the scale to guard against inconsistent responses. In addition, a signature was collected at the end of the scale, in which the participant answered each item on this scale honestly and to the best of my ability. Finally, instructions were provided at the beginning of the scale to inform the participant of how to fulfill the survey, remind them to read over each question carefully, and encourage honest participation. No items representing other NEO facets were used. The full scale can be found in the appendix of this paper. The other scales used in this study, the Hypercompetitive Attitude Scale by Ryckman and the MACH-IV, were slightly modified with the same instruction and signature as the agreeableness scale.

\section{Procedure}

After being randomly selected and completing their informed consent forms, participants were instructed to fill out the scales in a separate room following the conclusion of the camp. A proctor was present to answer any questions and maintain the integrity of the study. Participants were not allowed to communicate with one another while answering the surveys. They were also required to fill out a participant information form that collected personal data such as their name, age, and ethnic background. Once they completed their forms, they were dismissed and the data from their scales were scored and recorded. All-time US Squash rankings for each participant were procured through the US Squash website by searching their names and viewing their user profiles. All-time rankings were used as opposed to 
current rankings because of the detrimental impact the Covid-19 pandemic has had on the US Squash ranking system: rankings were skewed due to a lack of tournaments.

\section{Results}

This study was the first to examine the relationship between Machiavellianism, agreeableness, and hypercompetitiveness in adolescent squash athletes. In order to do so, a Pearson correlation was used to assess the correlation between the personality traits. The results revealed that Machiavellianism had a strong negative correlation with agreeable$\operatorname{ness}(\mathrm{r}=-0.678)$ with a $\mathrm{p}$-value of 0.000039 . This relationship was found to be statistically significant, as the $\mathrm{p}$-value was lower than the alpha-level of .05. Figure 1 portrays the correlation between Machiavellianism and agreeableness. Besides Machiavellianism, agreeableness was discovered to be negatively correlated with hypercompetitiveness $(\mathrm{r}=$ 0.690). The correlation between hypercompetitiveness and agreeableness was strong and statistically significant(pvalue $=0.000025)$ at an alpha-level of .05 . The relationship between agreeableness and hypercompetitiveness is displayed in Figure 2. Finally, hypercompetitiveness was found to have a moderate positive correlation with Machiavel$\operatorname{lianism}(\mathrm{r}=0.496)$. The $\mathrm{p}$-value was 0.005312 , meaning that the correlation was statistically significant at the alphalevel of 05 . Figure 3 features the correlation between hypercompetitiveness and Machiavellianism. The first hypothesis of this study was that there would be a strong positive correlation between hypercompetitiveness and Machiavellianism, and that both of these traits would share strong negative correlations with agreeableness. The results did not entirely support this hypothesis, as Machiavellianism and hypercompetitiveness only had a moderate positive correlation as opposed to a strong correlation.

\section{Correlation between Machiavellianism and Agreeableness}

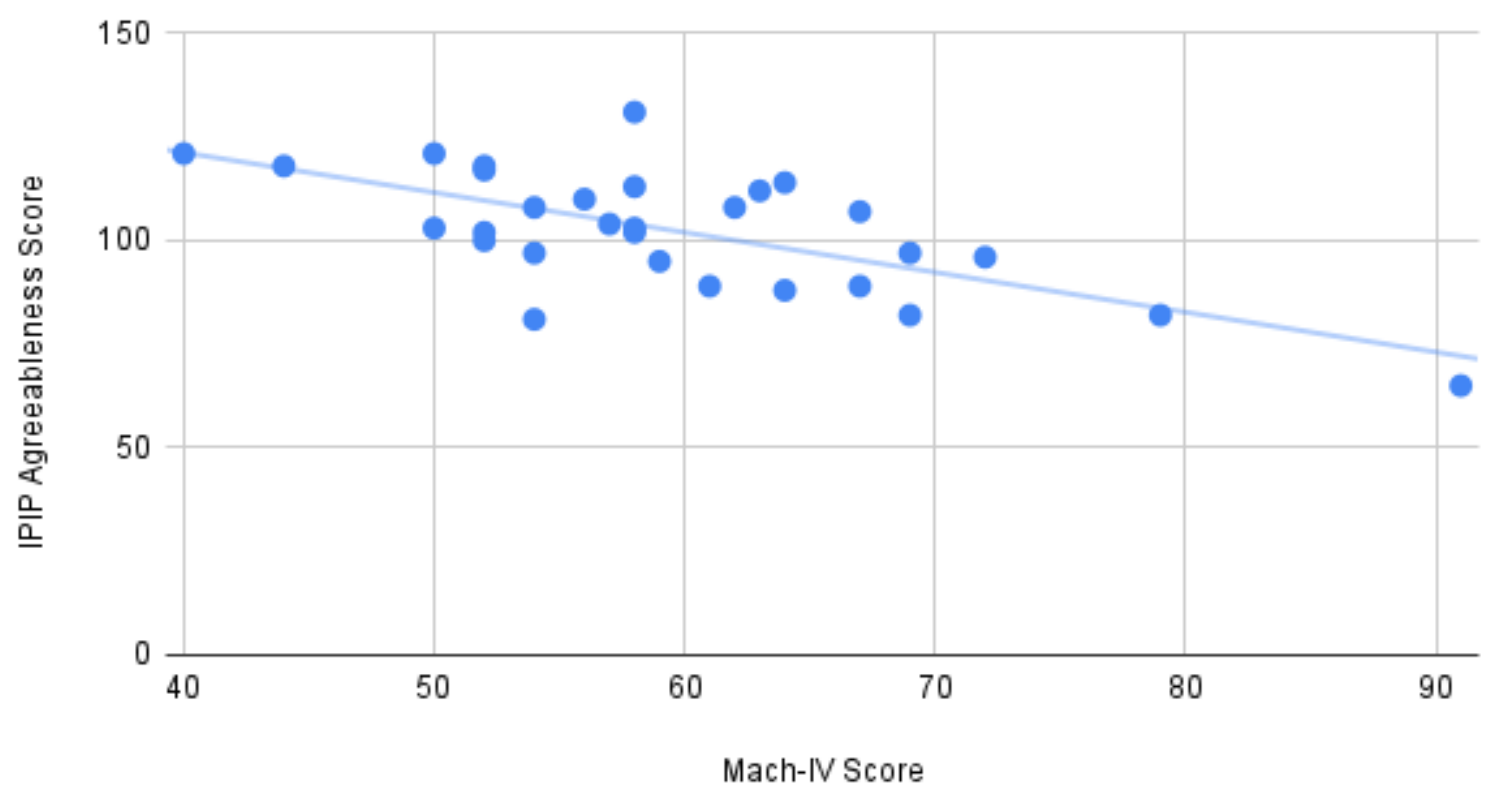

Figure 1. Scatterplot displaying the correlation between scores on the MACH-IV and the IPIP Agreeableness Meas$\operatorname{ure}(\mathrm{r}=-0.678, \mathrm{p}=0.000039)$. 


\section{Correlation between Hypercompetitiveness and Agreeableness}

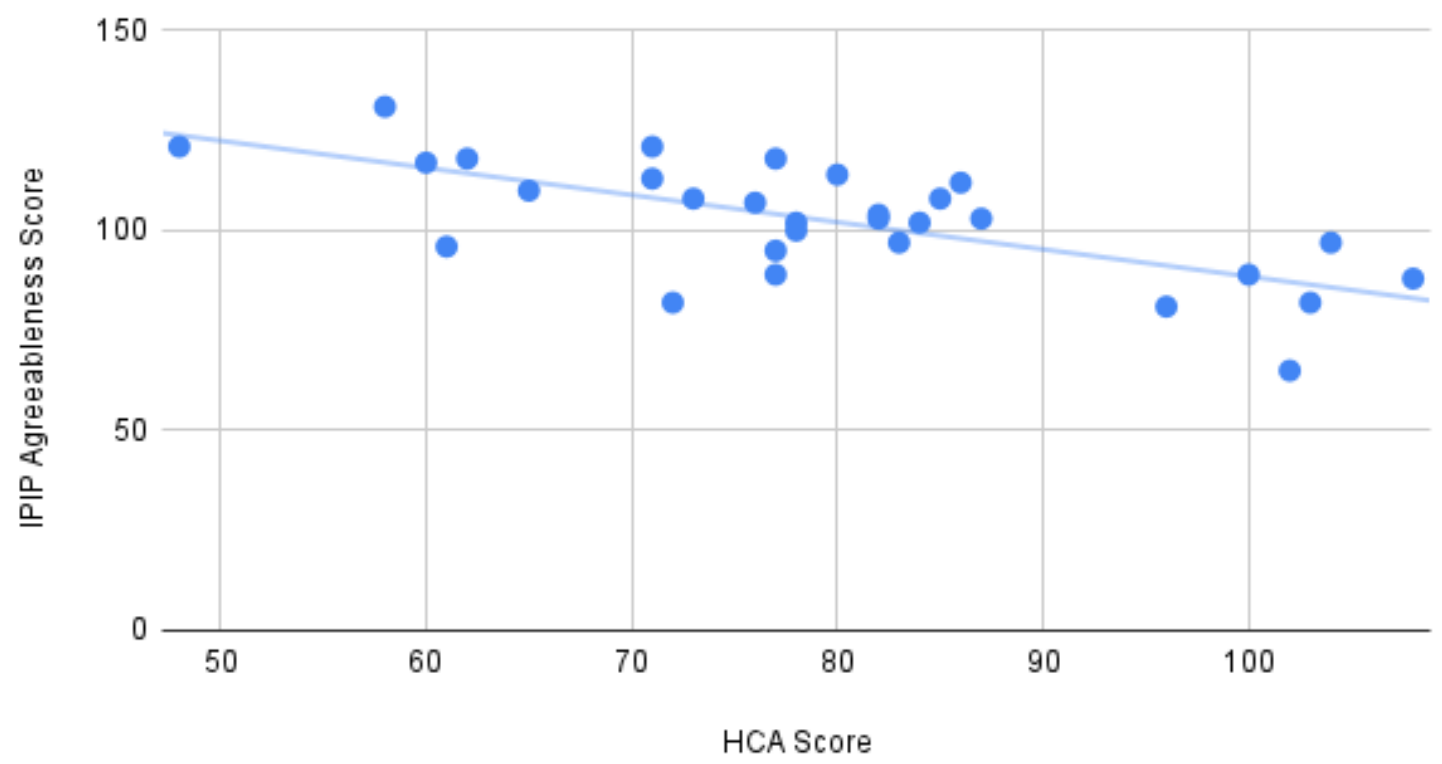

Figure 2. Scatterplot showing the relationship between scores on the HCA(Hypercompetitive Attitude scale) versus the IPIP Agreeableness Measure $(r=-0.690, p=0.000025)$.

\section{Correlation between Hypercompetitiveness and} Machiavellianism

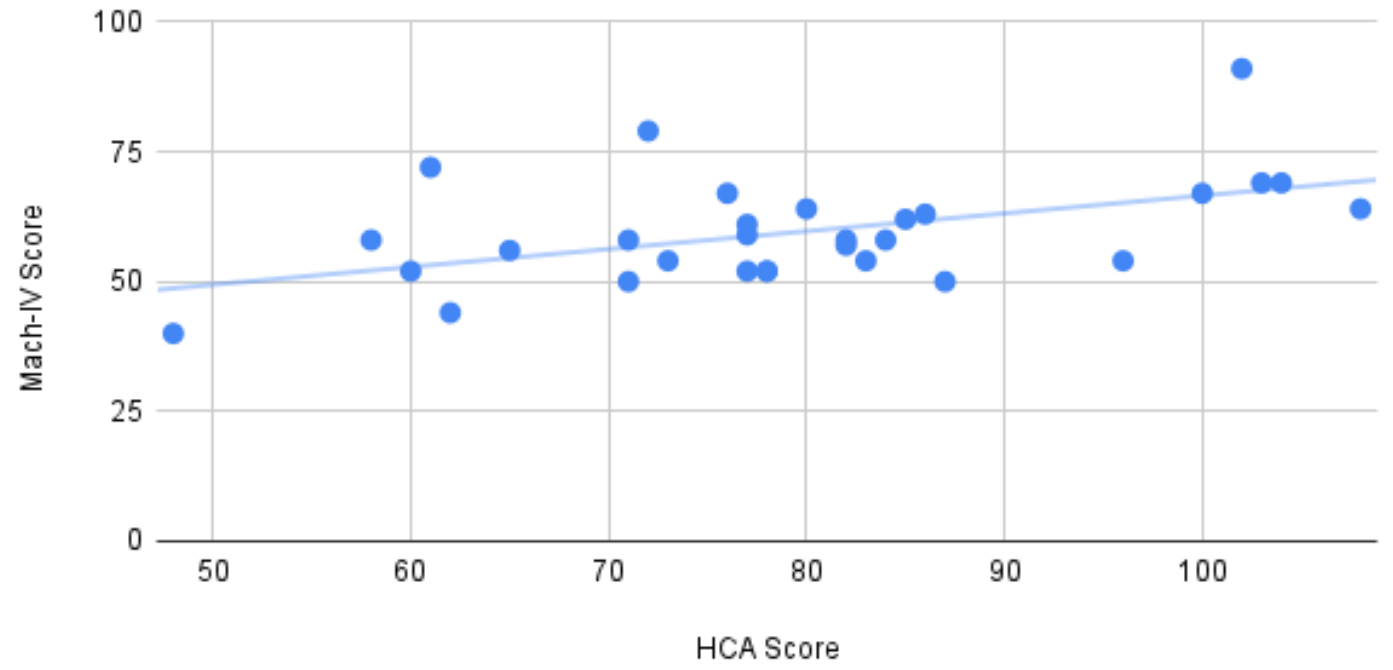

Figure 3. Scatterplot showing the correlation between HCA and MACH-IV scores( $r=0.496, p=0.005312)$. The correlation between the two data sets was expected to be stronger according to the hypothesis. 
The second purpose of this study was to determine whether those who have reached higher all-time US Squash national rankings would score higher in the traits of hypercompetitiveness and Machiavellianism. Based on the rankings of each participant, two groups were created and compared: high rankers $(\mathrm{N}=13)$ and low rankers $(\mathrm{N}=$ 17). High rankers were participants whose highest all-time national rankings were at least 15 in the nation, and low rankers were participants with national ranks below that threshold. There was an extremely wide range of all-time national rankings in the sample, from participants who were once ranked one in the nation to those ranked in the hundreds. To determine whether the mean scores in hypercompetitiveness and Machiavellianism of the two groups were significantly different, two independent t-tests were performed. When comparing hypercompetitiveness scores between high rankers(Mean= 85.00, $\mathrm{SD}=14.8099)$ and low rankers(Mean= 75.35, $\mathrm{SD}=13.6745)$, it was found that the difference in hypercompetitiveness was not statistically significant at an alpha-level of .05 with a T-value of 1.85 and a p-value of .075262. Similarly, when Machiavellianism scores were compared between high(Mean=62.62, $\mathrm{SD}=$ 10.5715) and low rankers(Mean= 57.18, $\mathrm{SD}=9.58019)$, there was no statistical difference, with a $\mathrm{T}$-value of 1.47 and a p-value of .151718. Therefore, the second hypothesis of this study was not supported.

\section{Discussion}

Contrary to the second hypothesis, there were no significant differences between high rankers and low rankers in hypercompetitiveness and Machiavellianism. This is the first study of this nature in that the actual rankings of adolescent participants were attempted to be explained by personality differences. It is possible that the differences in age divisions may be responsible for this result, as it is commonly asserted that the younger age divisions are significantly easier to climb than the older age divisions, either due to talent or hard work. Thus, it is possible that perhaps not as much competitiveness or cunning is required to achieve in the younger divisions versus the older ones. It is also possible that a larger random sample of US junior squash players would yield different results. One of the major limitations of this study was the fact that participants were only taken from college squash camps. The individuals who chose to participate in the camps may have done so for reasons other than wanting to compete against others, such as a desire to improve or connect with other players. Further research should take age divisions into account and involve a randomized sample from across the United States.

The first hypothesis, on the other hand, was far more supported by the results. Machiavellianism and hypercompetitiveness were found to have strong negative correlations to agreeableness, as the hypothesis predicted. It is no wonder that those who are characterized as "moving against others" by the trait of hypercompetitiveness are less likely to cooperate or get along with people. Machiavellianism and agreeableness have long been found to oppose one another. It is also not surprising, then, that the two traits were correlated negatively with one another: competitors who endorse deception "gamesmanship" practices are unlikely to hold any ostensible moral standards or display trust. These results demonstrate that these personality trends apply to adolescents as well, specifically adolescent athletes. However, Machiavellianism and hypercompetitiveness were only moderately correlated. This means that those who were "hungrier" for the win tended to be additionally willing to resort to unscrupulous methods to attain it. These results agree with previous research performed on adult athletes. Again, a larger sample size could potentially reveal a stronger correlation between these two traits.

\section{Limitations}

A potential limitation in this study was the fact that self-report measures were used in the collection of data. While the self-report scales were empirically validated and had mechanisms to ensure fair participation, it is still possible that participants may have figured out the reverse-scoring elements of the scale and chose to rate themselves based on either their own self-image or one that would be acceptable to society. These ratings would have been reflected in the 
results as higher scores in Agreeableness and lower scores in Machiavellianism and hypercompetitiveness. Participants who were especially Machiavellian would have been able to lie on the scales with ease. Another limitation that has already been stated was the sample size; a sample size of thirty participants is hardly adequate. A larger sample size could have produced results that were significantly different to those of this study. In addition, the language in the self-report measures may have been confusing to some participants. There were several participants who had to ask the instructor regarding several words in the items and the instructions(e.g, "envy"). Misunderstandings in the items or instructions of the scales may have resulted in less accurate results. Finally, a perhaps unavoidable limitation of this study was the regulations surrounding the US Squash ranking system. Given that many players have birthdays before tournaments with larger amounts of ranking points offered, it is possible that those players would have achieved higher ranks in their age division had they been eligible to play those tournaments.

\section{Future Research}

While this study is the first to examine adolescent individual-sport athletes, future research could seek to discover whether the same results would be replicated in a sample of adolescent team-sport athletes. Playing on a team, while competitive, requires an increased amount of cooperation and may yield different personality correlates and profiles. If this study were to be replicated, a larger sample size should be used with considerations to the varying age divisions. Another study that should be performed to lend credibility to the results from this study would use a different individual sport, such as fencing. Within a similar study, it would be interesting to discover the relationships between hypercompetitiveness and Machiavellianism with the other Big Five personality traits while also examining their relationship to the highest national rank. It is possible that there is an underlying trait that correlates with high athletic achievement and hypercompetitiveness(such as conscientiousness) that may not have been revealed in this study.

\section{Acknowledgments}

The author of this study would like to thank Avery Park for assisting in data collection and his advisor, Mr. Herman for providing good counsel.

\section{References}

Barrett, Ruth Shalit. "The Mad, Mad World of Niche Sports among Ivy League-Obsessed Parents." The Atlantic, Atlantic Media Company, 2 Nov. 2020, www.theatlantic.com/magazine/archive/2020/11/squash-lacrosse-nichesports-ivy-league-admissions/616474/.

Cherry, K. (2019, October 14). What Are the Big 5 Personality Traits? Verywell Mind. Retrieved 18 Aug 2021, from https://www.verywellmind.com/the-big-five-personality-dimensions-2795422

Christie, Richard, et al. Studies in Machiavellianism. Academic Press, 1970.

Cooper, S., \& Peterson, C. (1980). Machiavellianism and spontaneous cheating in competition. Journal of Research in Personality, 14(1), 70-75. https://doi.org/10.1016/0092-6566(80)90041-0

DeShong, H. L., Helle, A. C., Lengel, G. J., Meyer, N., \& Mullins-Sweatt, S. N. (2017). Facets of the Dark Triad: Utilizing the Five-Factor Model to describe Machiavellianism. Personality and Individual Differences, 105, 218223. https://doi.org/10.1016/j.paid.2016.09.053

González-Hernández J, Cuevas-Campos R, Tovar-Gálvez MI, Melguizo-Rodríguez L. Why Negative or Positive, If It Makes Me Win? Dark Personality in Spanish Competitive Athletes. Int J Environ Res Public Health. 2020 May 17;17(10):3504. doi: 10.3390/ijerph17103504. PMID: 32429570; PMCID: PMC7277204. 
Horney, Karen. The Collected Works OF KAREN Horney, v. 1: The Neurotic Personality of Our Time, New Ways in Psychoanalysis, and Our Inner CONFLICTS. W.W. Norton \& Company, 1937.

Houston, J. M., Queen, J. S., Cruz, N., Vlahov, R., \& Gosnell, M. (2015). Personality traits and winning:

Competitiveness, hypercompetitiveness, and Machiavellianism. North American Journal of Psychology, 17(1), 105-

112 .

Johnson, J. A. (2014). Measuring thirty facets of the Five Factor Model with a 120-item public domain inventory:

Development of the IPIP-NEO-120. Journal of Research in Personality, 51, 78-89.

Láng, András, Birkás, Béla.(2015). "Machiavellianism and Parental Attachment IN Adolescence: Effect of the

Relationship with Same-Sex Parents - András Láng, Béla BIRKÁS, 2015.” SAGE Journals, journals.sagepub.com/doi/full/10.1177/2158244015571639.

McCrae, Robert R., et al. "The NEO-PI-3: A More READABLE Revised Neo Personality Inventory." Journal of

Personality Assessment, vol. 84, no. 3, 2005, pp. 261-270., doi:10.1207/s15327752jpa8403_05.

Menesini, E, Tassi F, Nocetini, A. The competitive attitude scale(CAS): a multidimensional measure of competitiveness in adolescence. Journal of Psychology and Clinical Psychiatry. 2018; 9(3) 240-244.

Paulhus, D. L., \& Williams, K. M. (2002). The Dark Triad of personality: Narcissism, Machiavellianism and psychopathy. Journal of Research in Personality, 36(6), 556-563. https://doi.org/10.1016/S0092-6566(02)00505-6 Rogoza, Radosław, and Jan Cieciuch. "Dark Triad Traits and Their Structure: An Empirical Approach." Current Psychology, vol. 39, no. 4, 2018, pp. 1287-1302., doi:10.1007/s12144-018-9834-6.

Ross, S. R., Rausch, M. K., \& Canada, K. E. (2003). Competition and cooperation in the five-factor model: Individual differences in achievement orientation. Journal of Psychology, 137, 323-337.

doi:10.1080/00223980309600617

Ryckman, R.M., Thornton, B., \& Butler, J.C. (1994). Personality correlates of the Hypercompetitive Attitude Scale: Validity tests of Horney's Theory of Neurosis. Journal of Personality Assessment, 62, 84-94.

Ryckman, R.M., Hammer, Max. (1990) Construction of a Hypercompetitive Attitude Scale,Journal of Personality Assessment, 55:3-4, 630-639, DOI: 10.1080/00223891.1990.9674097

Siegel, D. J. (2015). Brainstorm. Tarcher/Putnam.

Soldz, Stephen, and George E. Vaillant. "The Big Five Personality Traits and the LIFE Course: A 45-Year Longitudinal Study.” Journal of Research in Personality, Academic Press, 25 May 2002,

www.sciencedirect.com/science/article/abs/pii/S0092656699922432.

Vinney, Cynthia. "Understanding the Big Five Personality Traits." ThoughtCo, www.thoughtco.com/big-fivepersonality-traits-4176097.

Yearick, K.A.(2018). COMPETITIVE PERSONALITY AND WORK OUTCOMES: A META-ANALYSIS AND SCALE DEVELOPMENT. 